\title{
Mirror Based IMU-Camera and Internal Camera Calibration
}

\author{
Ghazaleh Panahandeh, Dave Zachariah, and Magnus Jansson \\ KTH Royal Institute of Technology, ACCESS Linnaeus Center \\ Stockholm, Sweden \\ \{ghazaleh.panahandeh, dave.zachariah, magnus.jansson\}@ee.kth.se
}

\begin{abstract}
In this paper, a novel method for estimating the transformation between an inertial measurement unit (IMU) and a camera together with the intrinsic parameters of the camera is proposed. The method relies on images of reflected feature points in a planar mirror captured by an uncalibrated camera mounted with an IMU. It does not rely on using a fixed calibration pattern in front of the moving camera and the motion is not limited to be planar in front of the mirror. Instead, known feature points located on the camera body are tracked over the time to estimate the IMU-camera transformation and camera intrinsic parameters. A state-space model of the system is derived and then used as input to the Sigma-Point Kalman filter framework. Simulation results show accurate estimation of both IMU-camera translation and rotation parameters as well as the camera intrinsic parameters.
\end{abstract}

Keywords-Inertial measurement unit;camera; calibration; Sigma-Point Kalman filter;

\section{INTRODUCTION}

Inertial navigation systems (INS) use a combination of motion sensors and rotational sensors to provide the position, orientation, and velocity of moving objects. At the core of each INS there is an inertial measurement unit (IMU), which generally consists of three orthogonal gyroscopes and three orthogonal accelerometers. The integration of these two clusters that provide angular velocity and specific force, yields attitude and position. However, inertial navigation systems suffer from integration drift, small errors in the measurement of acceleration and angular velocity are integrated into progressively larger errors in velocity, which are compounded into still greater errors in position. Therefore, the position must be periodically corrected by input from complementary sensors. One of the most common alternative sensor is a radio receiver, such as a global positioning system (GPS) receiver. Furthermore, the integration of vision and inertial sensors is one alternative to the GPS for indoor as well as outdoor navigation when the GPS signals are not available or reliable. By proper combination of information from an INS and a vision sensor, the error growth can be reduced.

Kalman filtering in particular, provides a theoretical framework for combining information from various sensors. Aiding the INS with a vision sensor such as a camera requires sensor-to-sensor relative transformation to be known; disregarding such an offset in the system will introduce unmodeled biases that may grow over time. Currently, several calibration techniques have been proposed to determine the 6 degrees-of-freedom (DoF) transformation between the IMU and the camera coordinate frames; however, many of them are based on using a static checkerboard calibration pattern. For instance, in [1] relative IMU-camera rotation and translation were estimated separately; although, by separate calibration of rotation and translation correlation between the parameters are not considered. In [2], calibration parameters were estimated by the extended Kalman filter through tracking feature points of a calibration pattern; furthermore, the observability of the nonlinear system is studied by employing the observability rank condition. A similar nonlinear system is studied in [3], in the gray-box system identification framework.

Using the image of planar mirror reflection (IPMR) in [4], the camera to base frame transformation is determined by the maximum-likelihood estimation method. In fact, IPMR is a virtual view of a scene, acquired by a camera with a pose symmetric to the mirror plane. By studying the epipolar geometry of virtual views of IPMR in [5], it is shown the possibility of estimating the pose of the camera. However, the rigid displacement between the virtual views are restricted to be planar in order to use the epipolar geometry of the virtual images. In planar motion the rotational axis should be normal to the plane containing the direction of translation [6]. The above methods all require intrinsically calibrated cameras, which can be achieved using a fixed calibration pattern [7]. However, the availability of the IMU signal is one advantage of such system that can be address the problem of camera self-calibration. For instance, in [8], these parameters were estimated using homographies, on the assumption of known relative rotation from external sensor. The availability of the IMU signals together with the camera can be used to find the camera intrinsic parameters. For instance, the problem of camera calibration with known rotation was studied in [8] based on the estimation of the homographies and fundamental matrices.

In this paper, we introduce an algorithm for jointly estimating the 6-DoF IMU-camera coordinate transformation and the intrinsic camera parameters, using the virtual view of feature points located at known positions on the camera body obtained from a mirror. Hence, calibration is achieved without the need for a calibration pattern fixed in the 
navigation frame. An additional advantage of the method is that the assumption of planar camera motion can be relaxed. To estimate the calibration parameters, the SigmaPoint Kalman filtering approach is used [9].

\section{SYSTEM DESCRIPTION}

In the following sections scalars are denoted by lowercase letters $(s)$, vectors by bold letters (f), and matrices by bold capitals $(\mathbf{K})$. The goal of our proposed algorithm is to estimate the position and orientation of the camera coordinate frame $\{c\}$ relative to the IMU frame $\{b\}$, where the camera is rigidly mounted in the IMU body frame. In order to simplify the treatment with different coordinate frames, we assume the navigation frame $\{n\}$ is located in the center of the mirror coordinate frame. Additionally, we look at the problem of finding camera intrinsic parameters. The camera intrinsic matrix $\mathbf{K}$ is considered to be uppertriangular with parameters $k_{u}, k_{v}, s, p_{u}$, and $p_{v}$; where $k_{u}$ and $k_{v}$ are the magnifications in the two coordinate directions of the image, $s$ is a skew parameter corresponding to a skewing of the coordinate axes, and $p_{u}$ and $p_{v}$ are the coordinates of the principal point [10].

\section{A. Time evolution of the system}

In order to estimate parameters in the Sigma-Point Kalman filter framework, we first describe the total system state vector as

$$
\begin{aligned}
& \mathbf{x}=\left[\begin{array}{lll}
\mathbf{x}^{i n s^{\top}} & \mathbf{x}^{i m u c^{\top}} \quad \mathbf{x}^{c c \top}
\end{array}\right]^{\top} \in \mathbb{R}^{26}, \\
& \mathbf{x}^{i n s}=\left[\begin{array}{lllll}
\mathbf{p}_{b}^{n \top} & \mathbf{v}_{b}^{n \top} & \mathbf{q}_{b}^{n \top} & \mathbf{f}^{b^{\top}} & \omega^{b^{\top}}
\end{array}\right]^{\top} \\
& \mathbf{x}^{i m u c}=\left[\begin{array}{ll}
\mathbf{p}_{c}^{b^{\top}} & \mathbf{q}_{c}^{b^{\top}}
\end{array}\right]^{\top}, \quad \mathbf{x}^{c c}=\left[\begin{array}{lllll}
k_{u} & k_{v} & p_{u} & p_{v} & s
\end{array}\right]^{\top} .
\end{aligned}
$$

The position and velocity of the IMU in the navigation frame are denoted by $\mathbf{p}_{b}^{n}$ and $\mathbf{v}_{b}^{n}$, respectively. $\mathbf{q}_{b}^{n}$ is the unit quaternion representing the rotation from body frame to navigation frame, and $\mathbf{q}_{c}^{b}$ is the unit quaternion representing the rotation from camera frame to navigation frame, and finally $\mathbf{f}^{b}$ and $\omega^{b}$ are the bias vectors affecting the accelerometer and gyroscope measurements, respectively.

The time evolution of the INS state and the IMU-camera transformation [2] can be described by

$$
\begin{array}{rlrlrl}
\dot{\mathbf{q}}_{n}^{b}(t) & =\frac{1}{2} \Omega(\omega(t)) \mathbf{q}_{n}^{b}(t) & \text { where } & \Omega(\omega) & =\left[\begin{array}{cc}
-[\omega]_{\times} & \omega \\
-\omega^{\top} & 0
\end{array}\right] \\
\dot{\mathbf{p}}_{b}^{n}(t) & =\mathbf{v}_{b}^{n}(t) & \dot{\mathbf{v}}_{b}^{n}(t) & =\mathbf{a}^{n}(t) & \dot{\mathbf{f}}^{b}(t) & =\mathbf{n}_{\delta \mathbf{f}}(t) \\
\dot{\omega}^{b}(t) & =\mathbf{n}_{\delta \omega}(t) & \dot{\mathbf{p}}_{c}^{b}(t) & =\mathbf{0}_{3 \times 1} & \dot{\mathbf{q}}_{c}^{b}(t) & =\mathbf{0}_{3 \times 1}
\end{array}
$$

where $\omega(t)$ is the rotational velocity of the body frame and $[\cdot]_{\times}$denotes the skew-symmetric matrix representation of the cross product operation [11]. The IMU and gyroscope bias increments, $\mathbf{n}_{\delta \mathbf{f}}$ and $\mathbf{n}_{\delta \omega}$, are modeled as white Gaussian noises. The output signals of the accelerometer $\mathbf{f}_{m}$ and the gyroscope $\omega_{m}$ are modeled as

$$
\begin{aligned}
\mathbf{f}_{m}(t) & =\mathbf{R}_{n}^{b}(t)\left(\mathbf{a}^{n}(t)-\mathbf{g}^{n}\right)+\mathbf{f}^{b}(t)+\mathbf{n}_{\mathbf{f}}(t) \\
\omega_{m}(t) & =\omega(t)+\omega^{b}(t)+\mathbf{n}_{\omega}(t)
\end{aligned}
$$

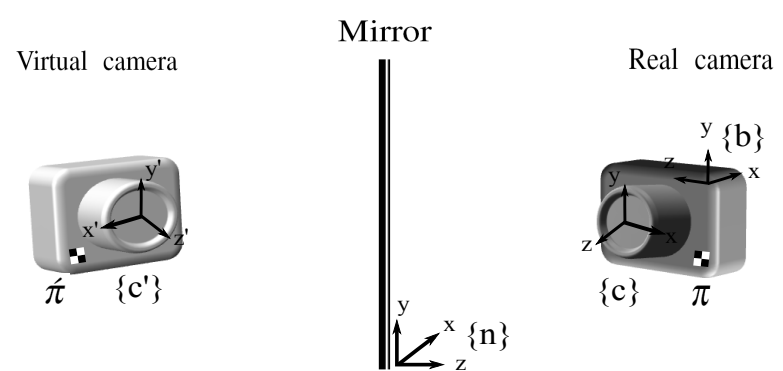

Figure 1: System sensor structure in front of the planar mirror

where $\mathbf{R}_{n}^{b}$ is the direction-cosine matrix which can be calculated from the corresponding quaternion [11], $\mathbf{n}_{\mathbf{f}}, \mathbf{n}_{\omega}$ are modeled as white Gaussian noises, and $\mathbf{g}^{n}$ is the gravitational acceleration expressed in the navigation frame.

\section{B. Discrete-time process model}

Using the standard additive error definition for the position, velocity, and biases $(\hat{x} \simeq x+\delta x)$ and quaternion error for the rotational angles $\psi\left(\delta \mathbf{q} \simeq\left[\begin{array}{ll}1 & \frac{\delta \psi}{2}\end{array}\right]^{\top}\right)$ (the advantage of quaternion error definition is the direct use of error angle vectors $\delta \theta$ and $\delta \varphi$ for the $\mathbf{q}_{b}^{n}$ and $\mathbf{q}_{c}^{b}$, respectively [11]), the discrete-time error state space model is given by the following equations:

$$
\begin{array}{rlrl}
\delta \mathbf{p}_{b, k+1}^{n} & =\delta \mathbf{p}_{b, k}^{n}+d t \delta \mathbf{v}_{b, k}^{n} \\
\delta \mathbf{v}_{b, k+1}^{n} & =\delta \mathbf{v}_{b, k}^{n}+d t\left[\hat{\mathbf{R}}_{b, k}^{n} \mathbf{f}_{m, k}\right]_{\times} \delta \theta_{k}+d t \hat{\mathbf{R}}_{b, k}^{n}\left(\delta \mathbf{f}_{k}^{b}+\mathbf{n}_{\mathbf{f}, k}\right) \\
\delta \theta_{k+1} & =\delta \theta_{k}-d t \hat{\mathbf{R}}_{b, k}^{n}\left(\delta \omega_{k}^{b}+\mathbf{n}_{\omega, k}\right) \\
\delta \mathbf{f}_{k+1}^{b} & =\delta \mathbf{f}_{k}^{b}+d t \mathbf{n}_{\delta \mathbf{f}, k} & \delta \omega_{k+1}^{b}=\delta \omega_{k}^{b}+d t \mathbf{n}_{\delta \omega, k} \\
\delta \mathbf{x}_{k+1}^{i m u c} & =\delta \mathbf{x}_{k}^{\text {imuc }} & \delta \mathbf{x}_{k+1}^{c c}=\delta \mathbf{x}_{k}^{c c}
\end{array}
$$

where $\hat{\mathbf{R}}_{b}^{n}$ is the estimated rotation matrix. According to the state vector model (1) and the discrete-time error state space model (4), the total error state vector is written as

$$
\begin{aligned}
\delta \mathbf{x} & =\left[\begin{array}{llllll}
\delta \mathbf{x}^{i n s} & \delta \mathbf{x}^{i m u c} & \delta \mathbf{x}^{c c \top}
\end{array}\right]^{\top} \in \mathbb{R}^{26}, \\
\delta \mathbf{x}^{i n s} & =\left[\begin{array}{llllll}
\delta \mathbf{p}_{b}^{n \top} & \delta \mathbf{v}_{b}^{n \top} & \delta \mathbf{q}_{b}^{n \top} & \delta \mathbf{f}^{\mathbf{b}^{\top}} & \delta \omega^{b^{\top}}
\end{array}\right]^{\top} \\
\delta \mathbf{x}^{i m u c} & =\left[\begin{array}{llllll}
\delta \mathbf{p}_{c}^{b^{\top}} & \delta \mathbf{q}_{c}^{b^{\top}}
\end{array}\right]^{\top}, \quad \delta \mathbf{x}^{c c}=\left[\begin{array}{lllll}
\delta k_{u} & \delta k_{v} & \delta p_{u} & \delta p_{v} & s
\end{array}\right]^{\top} .
\end{aligned}
$$

Hence, the state-space model of the discrete time process is given by

$$
\delta \mathbf{x}_{k+1}=f_{k}\left(\delta \mathbf{x}_{k}, \mathbf{n}_{k}\right) \in \mathbb{R}^{26}
$$

where the process noise $\mathbf{n}_{k}=\left[\begin{array}{llll}\mathbf{n}_{\mathbf{f}, k}^{\top} & \mathbf{n}_{\omega, k}^{\top} & \mathbf{n}_{\delta \mathbf{f}, k}^{\top} & \mathbf{n}_{\delta \omega, k}^{\top}\end{array}\right]^{\top}$ is assumed to be time invariant, with the covariance matrix $\mathbf{Q}$.

\section{Mirror reflection transformation}

To define the discrete time measurement model of our system, first of all, we need to derive the geometry of the reflected point in the planar mirror. For simplicity, we consider one single reflected feature point observed in the captured image. Without loss of generality, a standard righthanded Cartesian coordinate system is used for all the coordinate frames. The navigation frame, $\{n\}$, is located 
in the center of the mirror coordinate frame such that its $x y$ plane is the reflective surface see Figure 1 . Thus, the reflection of point $\pi$ is $\pi$. Using the unit vector along with the $z$ axis, $e_{\mathbf{z}}=[0,0,1]^{\top}$ it can be represented in the navigation frame as

$$
\dot{\pi}^{n}=\mathbf{A} \pi^{n} \in \mathbb{R}^{3}, \quad \text { where } \quad \mathbf{A}=\mathbf{I}_{3}-2 e_{\mathbf{z}} e_{\mathbf{z}}^{\top}
$$

and $\mathbf{I}_{3}$ is the identity matrix. Positions of feature point $\pi$ and its reflection $\pi$ with respect to the camera coordinate frame are $\pi^{c}$ and $\pi^{c}$, respectively as:

$$
\begin{aligned}
& \pi^{c}=\mathbf{R}_{n}^{c} \pi^{n}+\mathbf{p}_{n}^{c} \\
& \dot{\pi}^{c}=\mathbf{R}_{n}^{c} \hat{\pi}^{n}+\mathbf{p}_{n}^{c} .
\end{aligned}
$$

By substituting (7a) and (6) into (7b), we have

$$
\tilde{\pi}^{c}=\mathbf{R}_{n}^{c} \mathbf{A} \mathbf{R}_{n}^{c \top}\left(\pi^{c}-\mathbf{p}_{n}^{c}\right)+\mathbf{p}_{n}^{c} .
$$

Inserting the IMU-camera transformation, $\mathbf{R}_{n}^{c}=\mathbf{R}_{c}^{b^{\top}} \mathbf{R}_{n}^{b}$ and $\mathbf{p}_{c}^{n}=\mathbf{p}_{b}^{n}+\mathbf{R}_{n}^{b^{\top}} \mathbf{p}_{c}^{b}$, in (8) and using the fact that $\mathbf{p}_{c}^{n}=$ $-\mathbf{R}_{n}^{c \top} \mathbf{p}_{n}^{c}$, vector $\tilde{\pi}^{c}$ can be rewritten as

$$
\begin{aligned}
\tilde{\pi}^{c}= & \mathbf{R}_{c}^{b^{\top}} \mathbf{R}_{n}^{b} \mathbf{A}\left(\mathbf{R}_{c}^{b^{\top}} \mathbf{R}_{n}^{b}\right)^{\top} \pi^{c}-\mathbf{R}_{c}^{b^{\top}} \mathbf{R}_{n}^{b}\left(\mathbf{I}_{3}-\mathbf{A}\right) \mathbf{p}_{b}^{n} \\
& -\left(\mathbf{I}_{3}-\mathbf{R}_{c}^{b^{\top}} \mathbf{R}_{n}^{b} \mathbf{A}\left(\mathbf{R}_{c}^{b^{\top}} \mathbf{R}_{n}^{b}\right)^{\top}\right) \mathbf{R}_{c}^{b^{\top}} \mathbf{p}_{c}^{b} .
\end{aligned}
$$

Finally, by replacing A, (9) can be simplified to,

$$
\dot{\pi}^{c}=\pi^{c}-2 \mathbf{R}_{c}^{b^{\top}} \mathbf{R}_{n}^{b} e_{z} e_{z}^{\top}\left(\mathbf{R}_{n}^{b^{\top}} \mathbf{R}_{c}^{b} \pi^{c}+\mathbf{p}_{b}^{n}+\mathbf{R}_{n}^{b^{\top}} \mathbf{p}_{c}^{b}\right) .
$$

Equation (10) represents the position of the reflected feature point $\pi$ as a function of the known feature point position $\pi$ and the system state parameters (1). When the camera along with the IMU is moved in front of a planar mirror, the body frame angular velocity and specific force are measured by the IMU. Meanwhile, the camera records IPMRs. The projection of the reflected feature point $\pi^{c}$ to the image plane, based on the pinhole camera model [10], can be represented by

$$
\mathbf{z}=h\left(\mathbf{x}^{i n s}, \mathbf{x}^{i m u c}, \mathbf{x}^{c c}\right)+\mathbf{v}=\frac{\left[\begin{array}{ll}
\mathbf{I}_{2} & \mathbf{0}
\end{array}\right] \mathbf{K}}{\hat{\pi}_{z}^{c}}\left[\begin{array}{l}
\hat{\pi}_{x}^{c} \\
\hat{\pi}_{y}^{c} \\
\hat{\pi}_{z}^{c}
\end{array}\right]+\mathbf{v} \in \mathbb{R}^{2}
$$

where $\mathbf{v}$ is the feature-measurement noise with covariance matrix $\mathbf{R}=\sigma_{v}^{2} \mathbf{I}_{2}$. For $M$ observed reflected feature points, the discrete-time measurement model of the system is

$$
\delta \overline{\mathbf{z}}_{k}=h_{k}\left(\delta \mathbf{x}_{k}^{i n s}, \delta \mathbf{x}_{k}^{i m u c}, \delta \mathbf{x}_{k}^{c c}\right)+\mathbf{v}_{k} \in \mathbb{R}^{2 M} .
$$

\section{Sigma-Point Kalman Filtering}

Using the Sigma-Point Kalman Filter framework [9], the statistics of the random variables under the nonlinear process model (5) and measurement model (12) are calculated. Additionally, this technique removes the requirement to explicitly calculate Jacobians, which for complex functions, such as (10), can be a difficult task or numerically unstable. The high-rate IMU signal measurement propagates the state and the covariance matrix before a new measurement is received. From each new captured image the reflection of known feature points are detected, then the state estimates and the covariance matrices of the system are updated. An overview of the algorithm is given in Algorithm $1^{1}$.

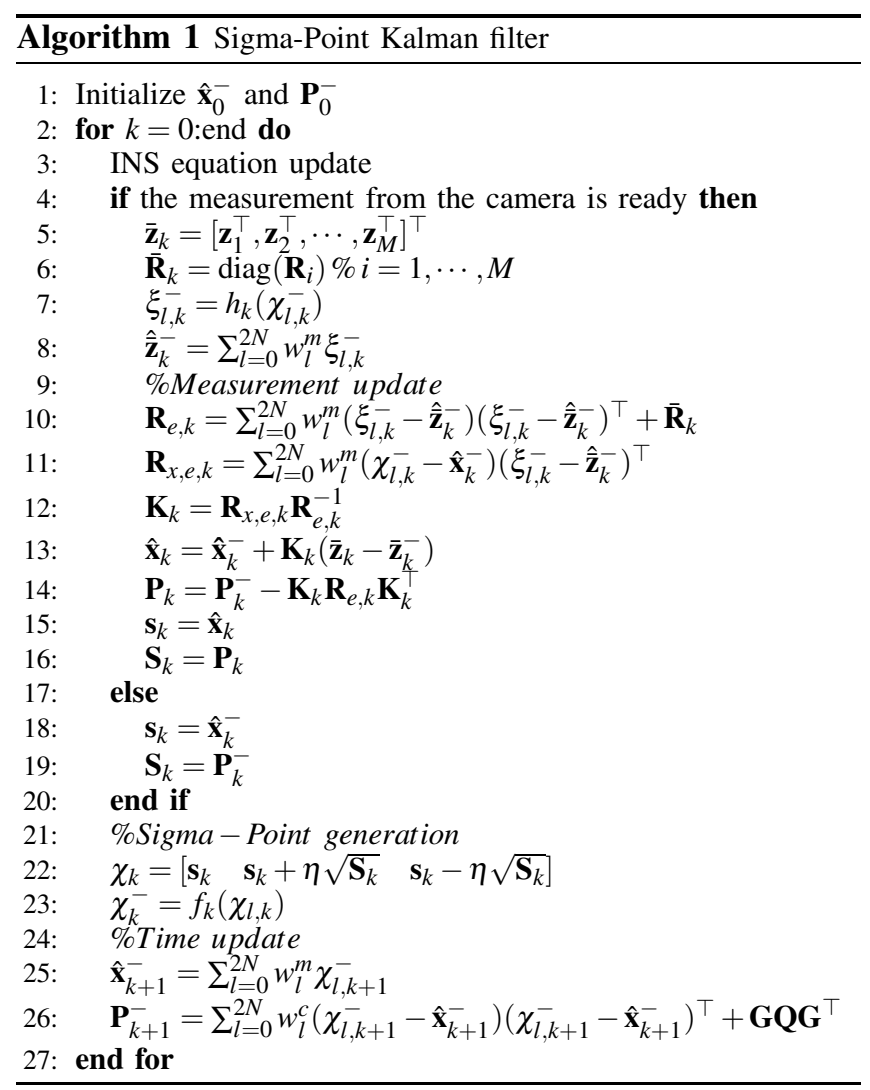

\section{Performance Evaluation}

The proposed calibration approach has been evaluated by Monte-Carlo simulations. The example IMU movement trajectory which is used for the performance evaluation of the estimators is shown in Figure 2. It displays a movement with roll $\phi \in\left[-90^{\circ}, 90^{\circ}\right]$ in a period of 12 seconds with a peak speed of about $0.4[\mathrm{~m} / \mathrm{s}]$ and angular rate of $2.9[\mathrm{rad} / \mathrm{s}]$. The sampling rate of the accelerometer and gyroscope output signals, $\mathbf{f}_{k}^{b}$ and $\omega_{k}^{b}$, is $100[\mathrm{~Hz}]$. The IMU accelerometer and gyros biases are set to $2 \cdot 10^{-3}\left[\mathrm{~m} / \mathrm{s}^{2}\right]$ and $5 \cdot 10^{-4}[\mathrm{rad} / \mathrm{s}]$ with the standard deviation of $6 \cdot 10^{-3}$ and $3 \cdot 10^{-3}$, respectively. The camera sampling rate is $10[\mathrm{~Hz}]$ and its intrinsic parameters are set to $k_{u}=k_{v}=833$ and $p_{u}=p_{v}=s=0$. The IMU-camera translation is $\mathbf{p}_{c}^{b}=[1,-5,10]^{\top}[\mathrm{cm}]$ and the rotation is set to $\mathbf{R}_{c}^{b}=\left[-90^{\circ}, 0^{\circ},-90^{\circ}\right]$. Our estimation method has been evaluated using 8 feature points placed in the camera body frame. In fact, at least 3 non-collinear points are necessary to be selected in the camera frame to define the camera coordinate frame [4]. A complete study

${ }^{1} \chi_{l}$ is the $l$-th column of the Sigma-Points matrix $\chi, N$ is the length of the state vector, $w_{l}^{c}, w_{l}^{m}$ are the weights of the SPKF [9], and $\mathbf{G}$ is the system noise propagation matrix. 
on feature points selection and pose estimation are given in [12].

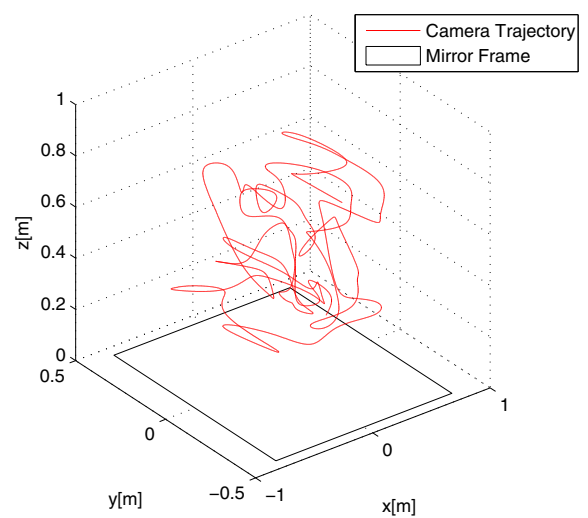

Figure 2: IMU trajectory over a $150 \times 90[\mathrm{~cm}]$ mirror, for 12 seconds.

1) Filter Initialization: The initial value of the error state estimates $\delta \hat{\mathbf{x}}$ are set to be zero. The standard deviations of the initial estimation error of the IMU position and the IMU-camera translation is set to $1 \cdot 10^{-2}[\mathrm{~m}]$ and for the velocity $1 \cdot 10^{-2}[\mathrm{~m} / \mathrm{s}]$. The standard deviations for the bias error estimates are set to $4 \cdot 10^{-2}\left[\mathrm{~m} / \mathrm{s}^{2}\right]$ and $1 \cdot 10^{-3}[\mathrm{rad} \cdot / \mathrm{s}]$, respectively. The value of the error state estimates in the attitude and IMU-camera rotation is initialized with $2\left[^{\circ}\right]$ and for the camera intrinsic parameters initial standard deviation of the error are set to 100 [pixel] for $k_{u}$ and $k_{v}$, and 1 [pixel] for $p_{u}, p_{v}, s$. To form $\hat{\mathbf{x}}_{0}^{-}$, actual initial errors were generated by a zero-mean Gaussian distribution with corresponding covariance matrix. The diagonal error covariance matrix $\mathbf{P}_{0}^{-}$ was initialized by the corresponding standard deviation.

2) Simulation Results: The estimated mean errors along with the root mean square error (RMSE) and $3 \sigma$-levels for the IMU-camera transformation and camera intrinsic parameters are plotted in Figure 3-4, respectively, over 12 seconds, using 100 Monte-Carlo simulations. The $3 \sigma$-levels are computed from the corresponding diagonal elements of filter's error covariance matrix that provide a representation of its estimate uncertainty. Zero-mean Gaussian noise with standard deviation of $\sigma_{v}=2$ is added to the projected image.

In addition, we investigated the effect of the level of the noise on the estimated values. We varied the noise level from 0.1 [pixel] to 2 [pixel] and for each noise level 100 Monte- Carlo simulations were performed. The mean of the estimated values for the intrinsic camera parameters are shown in Figure 5 for 12 records of the IMU and the camera data. It can be seen that the errors increase with pixel noise level, but the behavior is more robust for the $k_{u}$ and $k_{v}$.

Table I summarizes the final RMSE and the mean error of IMU-camera 6-DoF, transformation and rotation, and intrinsic camera calibration parameters for varying number of the observed reflected feature points in each image. It can be seen that increasing the number of feature points from 3 to 8 yield marginal improvement in the mean error of the parameters. The same simulation setting as described above is considered for this case and the image noise level is set to $\sigma_{v}=2$ [pixel]. Simulation results show that the proposed estimation method is able to reach subcentimeter and subdegree accuracy for the rotation and translation as well as subpixel errors for the intrinsic camera parameters.
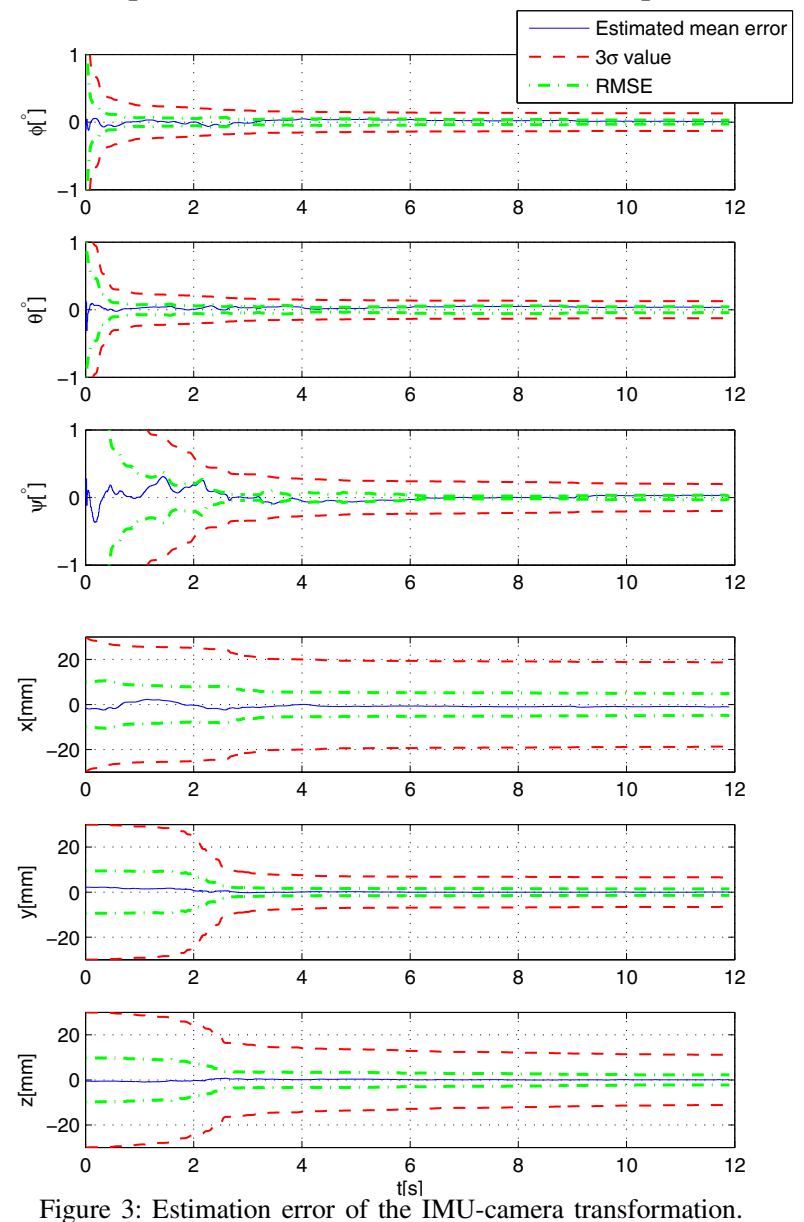

Figure 3: Estimation error of the IMU-camera transformation.

Table I: Error statistics, for 100 Monte-Carlo simulations.

\begin{tabular}{|l|c|c|c|c|c|c|}
\hline Feature points & \multicolumn{2}{|c|}{3} & \multicolumn{2}{|c|}{5} & \multicolumn{2}{c|}{8} \\
\hline Measure & RMSE & Mean & RMSE & Mean & RMSE & Mean \\
\hline \hline $\mathbf{p}_{c, x}^{b}$ & 6.5077 & -1.4314 & 5.4618 & -0.1900 & 5.0894 & -1.4499 \\
$\mathbf{p}_{c, y}^{b} \times 10^{-3}[\mathrm{~m}]$ & 2.2975 & -0.4743 & 1.8836 & -0.6266 & 1.6148 & -0.1795 \\
$\mathbf{p}_{c, z}^{b}$ & 2.7908 & -0.2778 & 2.6101 & -0.0674 & 2.0850 & -0.3298 \\
\hline$\varphi_{\phi}$ & 3.5631 & 0.6643 & 2.7283 & 0.3808 & 2.8427 & 0.5544 \\
$\varphi_{\theta} \times 10^{-2}\left[^{\circ}\right]$ & 4.5386 & 3.5479 & 4.4382 & 3.5479 & 4.3966 & 3.5121 \\
$\varphi_{\psi}$ & 2.1835 & 1.6777 & 2.0751 & 1.6993 & 2.3186 & 1.8207 \\
\hline $\mathbf{k}_{u}$ & 0.5154 & 0.3670 & 0.5517 & 0.3791 & 0.5565 & -0.3640 \\
$\mathbf{k}_{v}$ & 0.5886 & 0.4043 & 0.6230 & 0.4156 & 0.5954 & -0.4056 \\
$\mathbf{p}_{u}[\mathrm{pixel}]$ & 0.3629 & 0.6073 & 0.3896 & 0.59449 & 0.2884 & 0.3911 \\
$\mathbf{p}_{v}$ & 0.6133 & -0.0877 & 0.4613 & -0.0376 & 0.4796 & -0.0765 \\
$\mathbf{s}$ & 0.3616 & 0.2829 & 0.3460 & 0.2208 & 0.3168 & 0.1936 \\
\hline
\end{tabular}

\section{CONCLUSION}

An approach for estimating the 6-DoF IMU-camera transformation and, additionally, intrinsic camera parameters has 

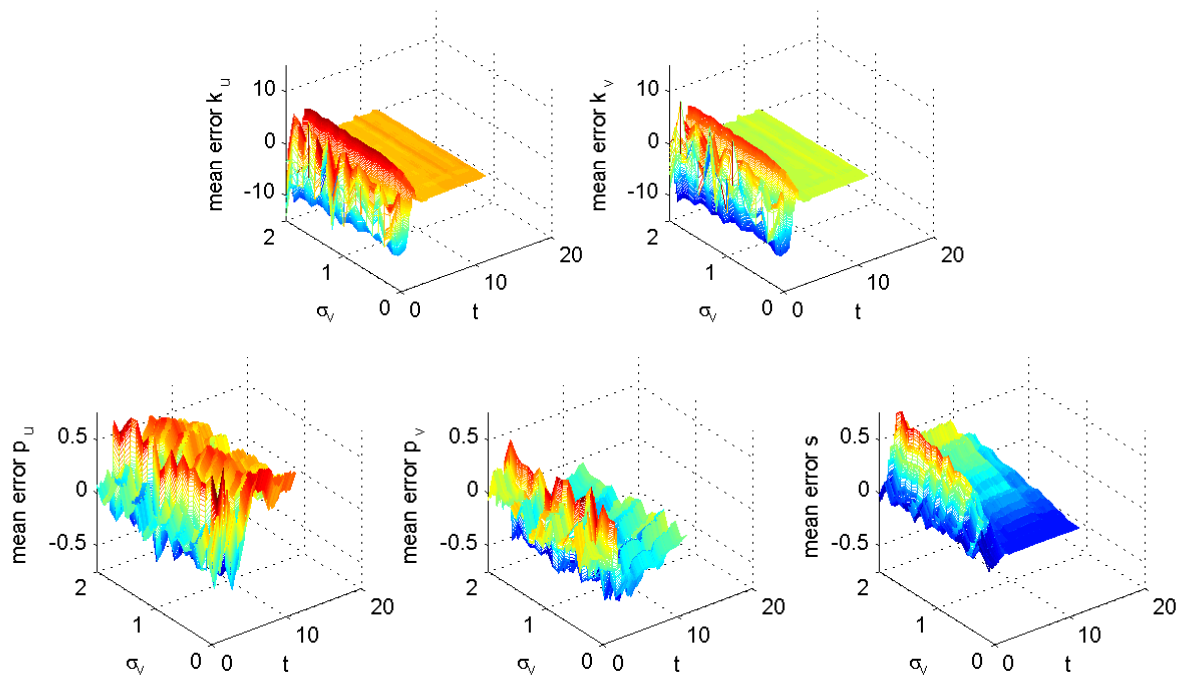

Figure 5: Image pixel noise robustness measurement for the mean error of camera intrinsic parameters.
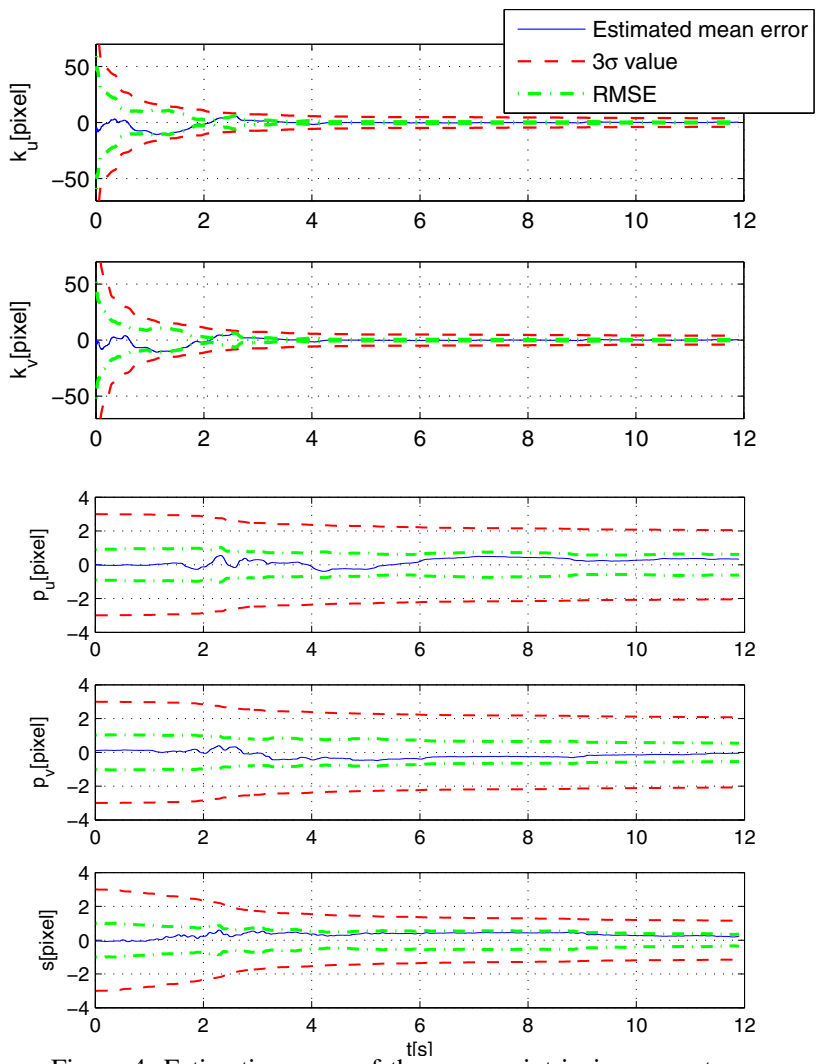

Figure 4: Estimation error of the camera intrinsic parameters.

been proposed. The method does not require a fixed calibration pattern. Instead, our calibration method is based on IPMR. Combining the image of the reflection with the IMU measurement, we show that it is possible to have a reliable estimate of the camera intrinsic as well as for IMUcamera calibration parameters. Moreover, no restriction of the IMU-camera movement in front of the planar mirror is considered. The problem of nonlinearity was addressed using the Sigma-Point Kalman filter. Simulation results show that the estimator has a good performance even with a small number of selected feature points and has a robust behavior to the noise pixel level in the image.

\section{REFERENCES}

[1] J. Lobo and J. Dias, "Vision and inertial sensor cooperation using gravity as a vertical reference," IEEE Trans. Pattern Anal. Mach. Intell., vol. 25, pp. 1597-1608, Dec. 2003.

[2] F. Mirzaei and S. Roumeliotis, "A kalman filter-based algorithm for imu-camera calibration," in Proc. of IROS, 2007.

[3] J. Hol, T. Schön, and F. Gustafsson, "Relative pose calibration of a spherical camera and an imu," in Proc. of 7th IEEE/ACM, ISMAR., pp. 21-24, 2008.

[4] J. A. Hesch, A. I. Mourikis, and S. I. Roumeliotis, "Mirrorbased extrinsic camera calibration," in The Eighth Int. Work. on the Algorithmic Foundations of Robotics, Dec. 2008.

[5] R. Rodrigues, J. a. P. Barreto, and U. Nunes, "Camera pose estimation using images of planar mirror reflections," in Proc. of the 11th European conference on Computer vision: Part IV, ECCV'10, pp. 382-395, Springer-Verlag, 2010.

[6] J. Gluckman and S. Nayar, "Planar catadioptric stereo: geometry and calibration," in Proc. of Computer Vision and Pattern Recognition. IEEE Computer Society Conference., 1999.

[7] J.-Y. Bouguet, "Camera calibration toolbox," Computation vision at Caltech, www.vision.caltech.edu/bouguetj/, 2008.

[8] J.-M. Frahm and R. Koch, "Camera calibration with known rotation," in Proc. of IEEE, ICCV, pp. 1418-1425, 2003.

[9] S. J. Julier and J. K. Uhlmann, "A new extension of the kalman filter to nonlinear systems," in Proc. of Signal Processing, Sensor fusion, and Target Recognition, vol. 4, pp. 182-193, Apr. 1997.

[10] R. I. Hartley and A. Zisserman, Multiple View Geometry in Computer Vision. Cambridge University Press, ISBN: 0521623049, 2000.

[11] J. A. Farrell and M. Barth, Global Positioning System, Inertial Navigation and Integration. McGraw-Hill Companies, 1999.

[12] G. Panahandeh and M. Jansson, "IMU-camera self-calibration using planar mirror reflection," in Int. Conf. on Indoor Positioning and Indoor Navigation (IPIN), 2011. 